\title{
Kinetics of magnetic flocculation. II: Flocculation of coarse particles
}

\author{
J J M Janssen, J J M Baltussen, A P van Gelder and \\ J A A J Perenboom
}

High Field Magnet Laboratory and Research Institute for Materials, University of Nijmegen, Toernooiveld, NL-6525 ED Nijmegen, The Netherlands

Received 1 August 1989, in final form 30 July 1990

\begin{abstract}
In theoretical studies of flocculation kinetics one usually assumes that the flocculation process reaches a steady state very rapidly. This assumption is invalid when a long-range attraction between the particles is present. In this paper we will show that such a situation occurs for magnetic flocculation, provided that the particles are not too small. A full mathematical description of such a process is very difficult. As an alternative, a modified version of von Smoluchowski's model for rapid flocculation is presented, which describes the qualitative features of our experimental observations of aqueous $\mathrm{Mn}_{2} \mathrm{O}_{3}$ colloids with a particle diameter of about $0.8 \mu \mathrm{m}$ quite well. In particular this model suggests that the deviations from the 'steady state' predictions increase very rapidly with increasing particle size, making these results almost always incorrect for many practical applications of magnetic flocculation.
\end{abstract}

\section{Introduction}

When a suspension of particles is exposed to an applied magnetic field, particle aggregation occurs when the magnetic attraction between the induced or permanent magnetic moments is strong enough to outweigh the stabilizing forces of electro-chemical origin [1]. This phenomenon can be used as a separation technique, e.g. by choosing the field strength and the other circumstances such that only one component of a mixture aggregates (flocculates) and settles. The timescale of such a process is obviously of essential importance for such applications, which makes flocculation kinetics a subject of both fundamental and practical interest. In colloid chemistry [2,3] and aerosol science [4] flocculation kinetics has been studied for about a century. The established flocculation models were formulated originally by von Smoluchowski [5] and Fuchs [6], and have been refined, extended and experimentally confirmed in later years [2-4]. In all cases however, the interparticle interactions considered were spherically symmetric. Solving the basic equation of these models in the presence of the anisotropic magnetic interaction between two permanent or induced magnetic moments (which depends on the angle between the interacting moments) is not trivial. In the preceding paper [7] we have presented a numerical approach to the solution of this problem, and have so been able to calculate the dependence of the so-called stability factor $W$ of the suspension on the applied magnetic field $B$. Observation of the magnetic flocculation of fine-particle (about $0.2 \mu \mathrm{m}$ diameter) $\mathrm{Mn}_{2} \mathrm{O}_{3}$ colloids [7] has corroborated the predictions from our calculations, most notably a $B^{-2 / 3}$ dependence of $W$ in the high-field limit. Here we present experimental results for the magnetic flocculation of similar colloids that contain coarser particles (about $0.8 \mu \mathrm{m}$ diameter). For these suspensions deviations from the theoretical predictions are observed, which become larger for increasing field strength. Using a modified version of von Smoluchowski's model for rapid flocculation we will demonstrate that these deviations result from the fact that the flocculation process does not reach a steady state almost immediately, as is assumed in the usual theoretical treatments (including our own numerical approach) [2-7]. Of particular practical importance is the conclusion that the 'steady state' result for the initial flocculation rate may underestimate the actual rate more than an order of magnitude for particles larger than a few $\mu \mathrm{m}$.

\section{Von Smoluchowski's theory of rapid flocculation}

The first mathematical analysis of the flocculation process has been given by von Smoluchowski in 1917 [5], for the case of so-called rapid flocculation. In this limiting case no stabilizing forces are present to prevent or slow down the particle aggregation due to van der Waals attraction. Von Smoluchowski describes a colloid as a system of identical spheres in a liquid. The interaction between the spheres is represented by an infinitely deep, square potential well of range $R$. When the centre-to-centre distance $r$ between two particles is 
larger than $R$, there is no interaction and each particle freely performs its Brownian motion. For $r \leqslant R$ the two particles flocculate immediately and irreversibly. Since the potential well is supposed to model the van der Waals force, $R$ is assumed to be only slightly larger than the actual particle diameter $d$. The initial stage of the flocculation process can be modelled by putting one particle at the origin of the coordinate system, and assuming that all other particles flocculate only with this central particle and not among themselves. Furthermore the flocculation centre is assumed to remain unchanged during the process, which means that the particles reaching it simply disappear [2-5]. The evolution of the particle number density $n$ is then governed by the following diffusion equation:

$$
\frac{\partial n}{\partial t}=-\nabla \cdot j
$$

where the particle flux $j$ is given by:

$$
j=-D_{12} \nabla n
$$

and $D_{12}$ is the relative diffusion coefficient, which can be approximated as twice the Einstein diffusion coefficient $D$ for a single particle. This relative coefficient is introduced to account for the Brownian motion of the central particle [2-5]. The boundary conditions are:

$$
\begin{array}{ll}
n(r)=n_{0} & \text { at } t=0 \\
n(R)=0 & \text { for } t>0 \\
n(x)=n_{0} & \text { for } t \geqslant 0 .
\end{array}
$$

The solution of the diffusion equation under these conditions is [2-5]:

$n(r, t)=n_{0}\left[1-\frac{R}{r}\left(1-\frac{2}{\sqrt{\pi}} \int_{0}^{(r-R) / 2 \sqrt{D t}} \mathrm{e}^{-x^{2}} \mathrm{~d} x\right)\right]$.

For $t \gg R^{2} / D$ the integral can be neglected, and a 'steady state' is reached:

$$
n(r)=n_{0}\left(1-\frac{R}{r}\right) .
$$

The flocculation rate is equal to the number of particles that reach the flocculation surface $r=R$ per second, and is given by the total flux $j(R)$ of particles diffusing through this surface:

$$
j(R)=\left.D_{12} R^{2} \int_{0}^{2 \pi} \mathrm{d} \varphi \int_{0}^{\pi} \sin \theta \mathrm{d} \theta(\nabla n)_{r}\right|_{r=R} .
$$

Substitution of (4) yields:

$$
j(R)=8 \pi D R n_{0}\left(1+\frac{R}{\sqrt{\pi D t}}\right) .
$$

For $t \gg R^{2} / \pi D$ the time-dependent term is negligible, and the flocculation rate becomes a time-independent number. Let us take the time $t_{\mathrm{s}}$ at which the two terms in (7) have equal magnitude as a measure for the timescale at which the second term is significant. Using the well known expression $D=k_{\mathrm{B}} T / 3 \pi \eta d$ we then find:

$$
t_{\mathrm{s}}=\frac{3 \eta d R^{2}}{k_{\mathrm{B}} T}
$$

where $\eta$ is the viscosity of the carrier fluid, $k_{\mathrm{B}}$ Boltzmann's constant and $T$ the absolute temperature. When the potential well is chosen to represent the van der Waals force then $R \simeq d$, and we find for an aqueous colloid at room temperature:

$$
t_{\mathrm{s}}=0.75 d^{3} \quad d \text { in } \mu \mathrm{m} .
$$

We thus see that $t_{\mathrm{s}}$ decreases rapidly to a few milliseconds for particles well in the colloidal size range. Under usual circumstances in colloid chemistry the steady state is thus reached very rapidly [2, 3], and many authors only consider this case. When the potential well is supposed to model a long range attraction however, $R$ will be significantly larger than $d$, and the time-dependent term in (7) may become important in practical situations. We will see below that the presence of a strong magnetic interaction leads to such a situation.

\section{A non-stationary model for magnetic flocculation}

The description of flocculation kinetics in the presence of interparticle interactions is based on an approach by Fuchs [6]. In this model the equation for the particle flux (equation (2)) is extended with a drift term that accounts for the particle drift due to these interactions $[2,3,6]$ :

$$
j=-D_{12}\left(\nabla n+n \nabla V(r) / k_{\mathrm{B}} T\right)
$$

where $V(r)$ denotes the total interaction energy for the interaction between a particle at $r$ and the flocculation centre. Up to now the equation that results when this expression is substituted in (1) has been solved only for the steady state $(\partial n / \partial t=0)$, in which the flocculation rate is a time-independent number. Indeed the general time-dependent case is a difficult mathematical problem, even for spherically symmetric interactions. In order to explain the time-dependent flocculation rates that we have observed for magnetic flocculation of relatively coarse particles at high fields (to be discused later), we propose to use a modified version of von Smoluchowski's time-dependent approach, in which now the magnetic interaction rather than the van der Waals interaction is represented by a square potential well of range $R$. The other interparticle interactions are neglected, because we are especially interested in the high field limit. where the magnetic interaction dominates.

Our task is now to find an adequate expression for the range $R$ of the potential well when this well is supposed to represent the magnetic dipole-dipole interaction between two paramagnetic particles. We 
have encountered such an expression when studying the steady state limit of (1) in the presence of a magnetic interaction, using variational calculus. The magnetic dipole-dipole interaction between paramagnetic particles can be written as:

$$
\frac{V_{\mathrm{M}}}{k_{\mathrm{B}} T}=-\frac{2}{\sqrt{3}} \frac{\beta^{2}}{r_{\mathrm{d}}^{3}} P_{2}(u)
$$

where $r_{d}=r / d, u=\cos \theta$ and $P_{2}(u)$ is the second-order Legendre polynomial. The dimensionless parameter $\beta$ is given by:

$$
\begin{aligned}
\beta & =\chi B d^{3 / 2} \sqrt{\frac{\sqrt{3} \pi}{144 \mu_{0} k_{\mathrm{B}} T}} \\
& =2728 \chi B d^{3 / 2} \quad d \text { in } \mu \mathrm{m} .
\end{aligned}
$$

$\chi$ is the particle's magnetic volume susceptibility. First we write the particle number density as:

$$
n(r, \theta)=n_{0} f(r, \theta) \exp \left(-V(r, \theta) / 2 k_{\mathrm{B}} T\right)
$$

where $f(r, \theta)$ is a dimensionless function, to be determined below. The boundary conditions for $f$ follow from the corresponding conditions for $n$ (equation (3)):

$$
\begin{aligned}
& f(d, \theta)=0 \\
& f(x, \theta)=1 .
\end{aligned}
$$

Substitution of (13) into (1) and (10) leads to the following, second-order differential equation for $f$ in the steady state $(\partial n / \partial t=0)$ :

$$
\Delta f-\Psi(r, \theta) f=0
$$

where $\Psi(r, \theta)$ is defined as:

$$
\Psi(r, \theta)=\left(\nabla V / k_{\mathrm{B}} T\right)^{2} / 4-\Delta \mathrm{V} / 2 k_{\mathrm{B}} T
$$

For the present case, in which we only consider the magnetic interaction, we obtain:

$$
\Psi(r, \theta)=\frac{3 \beta^{4}}{4 d^{2} r_{\mathrm{d}}^{8}}\left(5 \cos ^{4} \theta-2 \cos ^{2} \theta+1\right) .
$$

In the preceding paper [7] we have solved (15) numerically, and obtained the relation $W=$ $3.014678(5) \times \beta^{-2 / 3}$ for the high-field limit. Here we will derive an approximate analytical expression for $f$ using the theory of variational calculus [8], which states that a function $f$ that satisfies the second-order differential equation (15) will also give the following integral an extremal value:

$$
F=\int \mathrm{d}^{3} r\left[(\nabla f)^{2}+\Psi f^{2}\right]
$$

In most situations of physical interest this extremum will be a minimum, but maxima and saddle points are possible too [8].
The stability factor $W$ of a colloid indicates how the flux $J$ through the capturing surface is changed compared to steady-state rapid flocculation:

$$
J=\frac{8 \pi D d n_{0}}{W} .
$$

For the exact form of $f$ it can be shown that the integral $F$ is related to the stability factor $W$ as:

$$
W=8 \pi d / F \text {. }
$$

For approximate expressions for $f,(20)$ can be interpreted as the definition of the approximate stability factor that corresponds to this approximation of $f$.

Since the numerical calculations have revealed that the exact $f$ is nearly isotropic when the magnetic interaction dominates [7], we choose an isotropic trial function here, which is inspired by the exact solution for $\Psi=0$ (equation (5)):

$$
f= \begin{cases}\left(1-c / r_{\mathrm{d}}\right)^{p} & \text { for } r_{\mathrm{d}}>c \\ 0 & \text { for } r_{\mathrm{d}}<c .\end{cases}
$$

Here $c$ and $p$ are adjustable parameters. Straightforward calculations now lead to:

$$
F(c, p)=4 \pi p^{2} c d I_{1}(p)+\frac{4 \pi \beta^{4} d}{c^{5}} I_{2}(p)
$$

where:

$$
\begin{aligned}
& I_{1}(p)=\int_{0}^{1} \mathrm{~d} y(1-y)^{2 p-2} \\
& I_{2}(p)=\int_{0}^{1} \mathrm{~d} y y^{4}(1-y)^{2 p} .
\end{aligned}
$$

The condition $\partial F / \partial c=0$ gives:

$$
c=\left(\frac{5 I_{2}}{p^{2} I_{1}}\right)^{1 / 6} \beta^{2 / 3} \text {. }
$$

Since $c$ has to be larger than or equal to unity, this expression also gives a lower bound for $\beta$. For smaller values of $\beta$ the expression for $f$ that has been chosen no longer obeys the boundary conditions.

Substitution of (25) in (22) reduces $F$ to a function of $p$ only, the minimum of which is numerically determined to occur for $p=1.60$, leading to:

$$
\begin{aligned}
c & =0.4843 \beta^{2 / 3} \\
F & =8.497 \mathrm{~d} \beta^{2 / 3} .
\end{aligned}
$$

Using the relation $W=8 \pi d / F$ we then find $W=$ $2.958 \beta^{-2 / 3}$. The variational calculus thus reproduces the exact result within two per cent, which suggests that the trial function defined in (21) gives an adequate approximation of the actual situation. The range $R$ of the square potential well (that is, the position of the spherical surface at which the particle number density vanishes because of capture) is now field-dependent, and is given by $c d=0.4843 d \beta^{2 / 3}$.

In our experiments we study the time dependence of the extinction during the first $40 \mathrm{~s}$, for light that is 
incident along the magnetic field. In the preceding paper [7] we have shown that the transmitted intensity $I$ is initially related to the number density of single particles $n_{1}$ as:

$$
\ln I(t)=K_{1}+K_{2} n_{1}(t)
$$

where:

$$
\begin{gathered}
K_{1}=\ln I_{0}-L C_{2} n_{1}(0) / 2 \\
K_{2}=-L\left(C_{1}-C_{2} / 2\right) .
\end{gathered}
$$

$I_{0}$ is the incident intensity, $L$ the distance the light travels through the colloid, and $C_{i}$ the extinction coefficient of an $i$-fold aggregate. In von Smoluchowski's model the number of single particles that is aggregating into doublets per second, is equal to $n_{0}$ times the particle flux towards the surface of a single central particle, given by (7). The number density for the remaining single particles is then:

$$
n_{1}(t)=n_{0}^{2}-8 \pi D R n_{0}^{2}\left(t+2 R \sqrt{\frac{t}{\pi D}}\right) .
$$

Substitution of this relation in (28) yields:

$$
\ln I(t)-\ln I(0)=R K_{2}^{\prime}\left(t+2 R \sqrt{\frac{t}{\pi D}}\right)
$$

where $K_{2}^{\prime}=8 \pi n_{0}^{2} D L\left(C_{1}-C_{2} / 2\right)$. This expression is valid in the early stages of the flocculation process, when aggregates containing more than two particles are rare. We now replace $R$ in (32) by the variational calculus parameter $c d$, and write:

$$
R=9.45 \times 10^{7} d^{2}(\chi B)^{2 / 3} .
$$

For $\mathrm{Mn}_{2} \mathrm{O}_{3}\left(\chi=5.3 \times 10^{-3}\right)$ we have

$$
\frac{R}{\sqrt{\pi D}}=2.47 d^{5 / 2} B^{2 / 3} \quad d \text { in } \mu \mathrm{m}
$$

If we now take the condition $R / \sqrt{\pi D t_{\mathrm{s}}}=1$ to give a measure of the timescale at which the square root term in the flux is relevant, we find:

$$
t_{\mathrm{s}}=6.1 d^{5} B^{4 / 3} \quad d \text { in } \mu \mathrm{m} .
$$

For $d=0.2 \mu \mathrm{m}$ at $15 \mathrm{~T}$ this yields only about $70 \mathrm{~ms}$, and the effect is unobservable in the measurements on fine-particle colloids [7]. For $d=0.8 \mu \mathrm{m}$ however, $t_{\mathrm{s}}$ is already about $2 \mathrm{~s}$ at a field of only $1 \mathrm{~T}$, and about $74 \mathrm{~s}$ at $15 \mathrm{~T}$. For the coarse-particle colloids used in the present work (particle diameter estimated by observation with an optical microscope to lie between 0.5 and $1 \mu \mathrm{m}$ ), the effect is indeed significant on the timescale of our experiments.

Substitution of (33) in (32) gives a relation for the evolution of the transmitted intensity as the sum of a linear and square-root term, with coefficients that depend on $B^{2 / 3}$ and $B^{4 / 3}$ respectively. For the actual analysis of our experiments with coarse particles, this expression has to be slightly modified, because the precise determination of the $t=0$ point turned out to be ambiguous in the actual experimental conditions. The travel of the sample to the centre of the magnet, and the shock at its arrival produce fluctuations in the initial signal for these large particles. The influence of the stray magnetic field, and the inertia of these particles may be among the reasons for this behaviour. We therefore introduce the $t=0$ point and the initial intensity as additional parameters, and rewrite (32) as:

$$
\ln I(t)=A_{1}\left(t-t_{0}\right)+A_{2} \sqrt{t-t_{0}}+A_{3}
$$

in which for $\mathrm{Mn}_{2} \mathrm{O}_{3}$ :

$$
\begin{aligned}
& A_{1}=2.87 \times 10^{-6} K_{2}^{\prime} d^{2} B^{2 / 3} \\
& A_{2}=4.94 A_{1} d^{5 / 2} B^{2 / 3} \quad d \text { in } \mu \mathrm{m} \\
& A_{3}=\ln I(0) .
\end{aligned}
$$

It should be noted however, that the parameters $A_{1}$ and $A_{2}$ are related through (38). When a suitable value of the particle diameter $d$ is chosen, this relation is fully determined. Thus $A_{1}$ is the only parameter that determines the shape of the curve, whilst the parameters $A_{3}$ and $t_{0}$ merely shift it relative to the axes.

\section{Experimental results and discussion}

The colloids used in the experiments have been prepared by dispersion of commercially available mineral powder in aqueous solutions of various amounts of $\mathrm{Na}_{4} \mathrm{P}_{2} \mathrm{O}_{7} \cdot 7 \mathrm{H}_{2} \mathrm{O}$ (typically 1 or $2 \mathrm{~g} \mathrm{l}^{-1}$ ). The size distribution in such colloids is rather broad. In principle, filtration through membranes of well defined pore size could have been used to narrow this distribution, but this is a tedious and time-consuming procedure for the preparation of sufficient quantities of colloid of a high enough concentration. In our work, we have made a distinction only between coarse-particle colloids, in which the constituent particles are microscopically observable, and fine-particle colloids, that are too small for such an observation [7]. In order to prevent a decrease in the average particle size as a result of gravitational settling, the coarse-particle colloids were used fairly soon after their preparation. In some cases the stability was somewhat reduced through addition of $\mathrm{KCl}$, which decreases the electric double layer repulsion $[2,3]$. Details of the experimental set-up have been described elsewhere [7]. Here we just mention that the sample is kept outside the magnet until this has reached the desired field strength, and is then moved to the magnet centre within a fraction of a second. In this way the magnetic interaction can be considered as switched on instantaneously up to the highest fields used.

Unlike the case of fine-particle colloids [7] the initial part of the $I(t)$ versus $t$ curves is not a straight line. Figure 1 shows some examples of the transmitted intensity as a function of time for a dilute coarse particle colloid (particle diameter estimated by observation with an optical microscope to lie between 0.5 and $1 \mu \mathrm{m})$. Some curves did tend towards a linear 

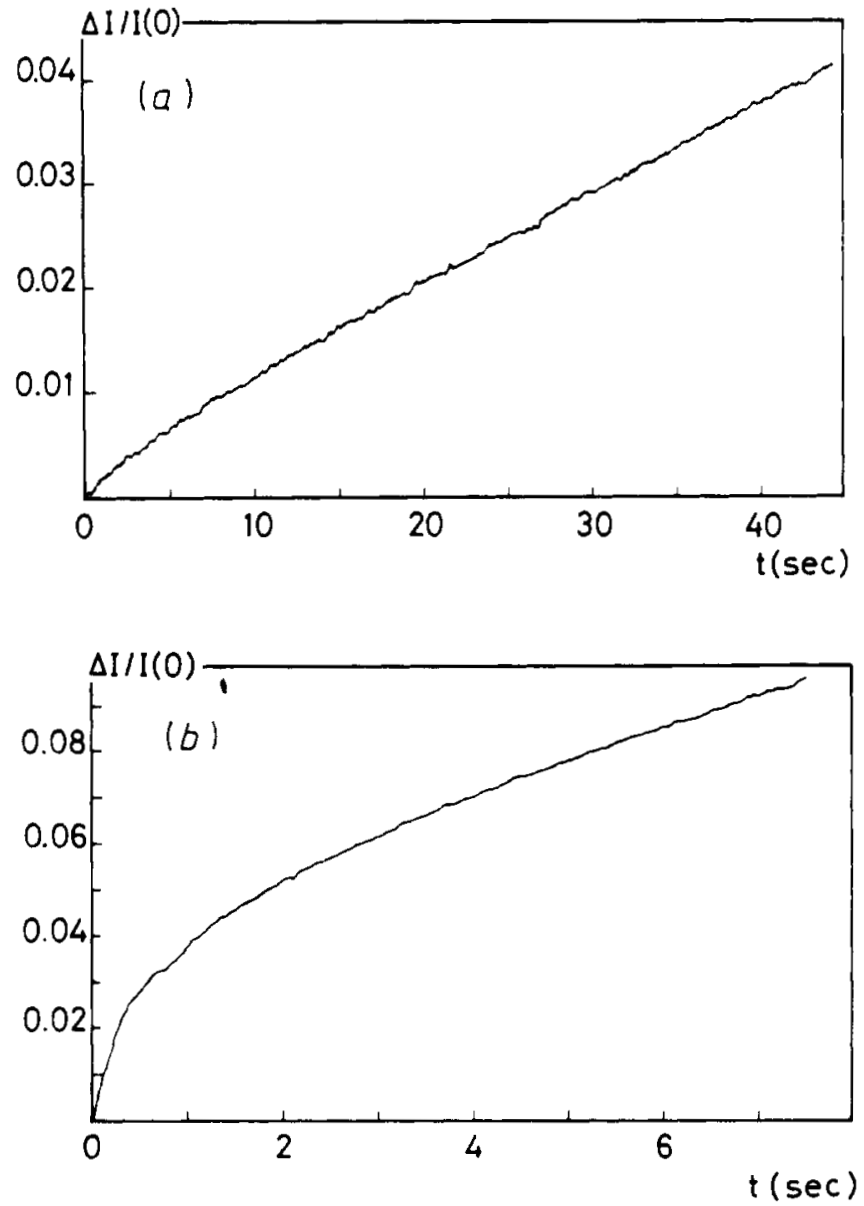

Figure 1. Typical example of the relative change in transmission through a coarse particle $\mathrm{Mn}_{2} \mathrm{O}_{3}$ colloid as a function of time, for applied magnetic fields of 1 and $12 \mathrm{~T}$ respectively.

behaviour after some time, but the initial part of the plots remained non-linear. In particular we noticed that the curvature of the initial part increased with increasing field strength.

A fit of the transmitted intensity to (36) is only allowed as long as the number of aggregates that contain more than two particles is negligible. Since we cannot judge a priori how far the validity of (36) goes, we have performed fits for several time intervals $\left[0, t^{\prime}\right]$, where $t^{\prime}$ has been varied between 5 and $40 \mathrm{~s}$. As long as this time interval lies within the validity range of (36), the fit parameters should remain essentially the same. Furthermore $t_{0}$ should fall within the actual inaccuracy of the $t=0$ point, which means that it should be smaller than a second. An example of such a fit is given in figure 2. Although the actual colloids are polydisperse, the quality of the fits turns out to be sensitive to the choice of the particle diameter $d$ in (38). The value $d=0.8 \mu \mathrm{m}$ given for figure 2 is the optimal choice within $0.1 \mu \mathrm{m}$, and is consistent with the microscopical estimate $(0.5 \mu \mathrm{m}<d<1 \mu \mathrm{m})$. For all coarse-particle colloids used in our experiments the evolution of the transmitted intensity could be described quite well as the sum of a linear and a squareroot term, with coefficients that are proportional to

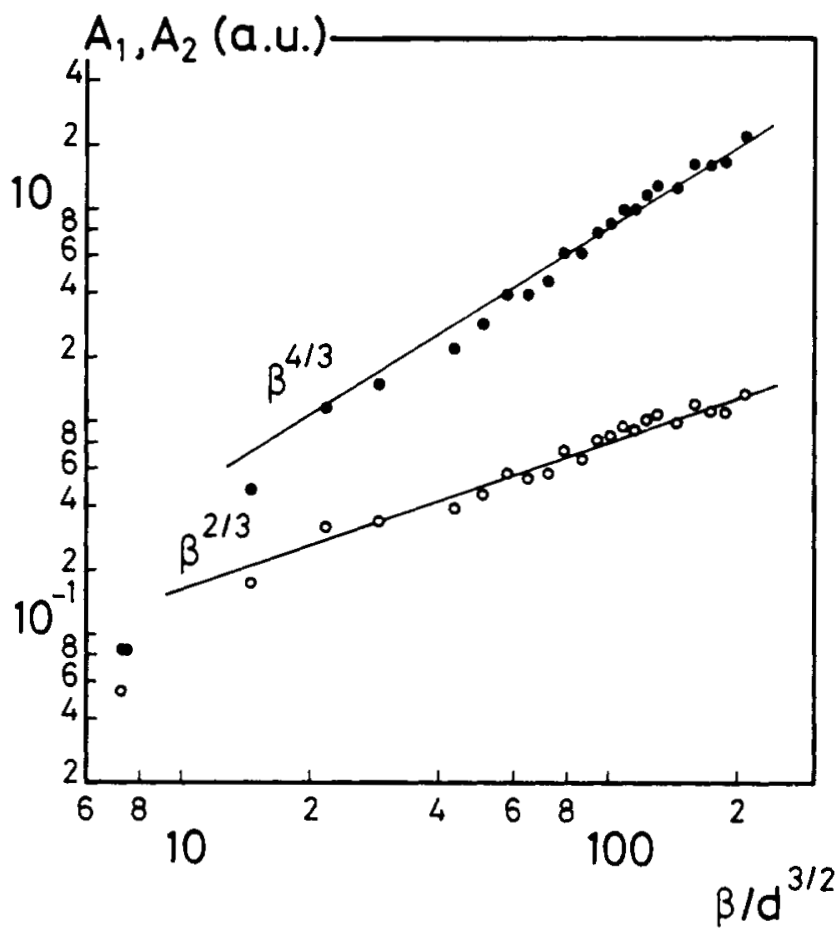

Figure 2. Fit parameters $A_{1}, A_{2}$ for the time-dependence of the transmission through a coarse-particle $\mathrm{Mn}_{2} \mathrm{O}_{3}$ colloid $(d=0.8 \mu \mathrm{m})$ as in equation (36), as a function of $\beta / d^{3 / 2}$.

$B^{2 / 3}$ and $B^{4 / 3}$ respectively. We thus conclude that our interpretation of the observed deviations from the 'steady state' calculations [7] is qualitatively correct. In future experiments the quantitative agreement between (36) and the experiments may be checked with better-defined colloids, although one should note that this equation results from a simplified model of magnetic flocculation. Lacking a full theoretical description (a non-stationary Fuchs model that includes the magnetic interaction) however, the model presented in section 3 may provide a basis for an approximative theory that can give useful estimates for the flocculation rate in practical situations.

The practical importance of the time-dependent term in the particle flux can be illustrated by comparing Svoboda's steady state analysis of the magnetic flocculation kinetics of $\mu \mathrm{m}$-sized mineral particles [1] with the predictions from our model. Svoboda calculates the time $T_{1 / 2}$ at which the total particle number is halved for specific examples of aqueous suspensions of hematite and siderite with a volume fraction $\varphi$ of 0.1 , using the expression for $T_{1 / 2}$ that has been derived by von Smoluchowski [5] under the assumption that the flocculation process is in a steady state:

$$
T_{1 / 2}=\left(4 \pi D R n_{0}\right)^{-1} \text {. }
$$

In the light of the results of the preceding sections however, it can be shown that the basic assumptions made in Svoboda's work are actually invalid. Equation (40) can be used only when the time-dependent term in (7) is negligible. If we again take the time $t_{\mathrm{s}}=R^{2}$ $\pi D$, at which the constant term and the time-dependent term in (7) have equal magnitude, as a measure 
for the time scale at which the $1 / \sqrt{t}$ term is relevant, we should have $t_{\mathrm{s}} \ll T_{1 / 2}$. Using the fact that the volume fraction $\varphi$ of a monodisperse colloid is given by $\varphi=$ $n_{0} \pi d^{3} / 6$ this leads to:

$$
\frac{24}{\pi} R_{\mathrm{d}}^{3} \varphi \ll 1 .
$$

In von Smoluchowski's model $R_{\mathrm{d}} \simeq 1$, so $\varphi$ should be much smaller than $\pi / 24 \simeq 0.13$. We thus see that even in ordinary rapid flocculation the steady state assumption is questionable for suspensions with a volume fraction of 0.1 , as considered by Svoboda.

In magnetic flocculation we have from equation (33) for hematite $\left(\chi \simeq 1.3 \times 10^{-3} \dagger\right)$ :

$$
R_{\mathrm{d}}=1.1 d B^{2 / 3} \quad d \text { in } \mu \mathrm{m}
$$

Svoboda calculates the stability factor $W$ for the socalled critical flocculation field [1], defined as the fieldvalue at which the maximum of the interaction energy as a function of the interparticle distance is zero, for a particle pair that is oriented along the applied field. For this field-value $\left(B \simeq 0.062 \mathrm{~T}\right.$ [1]) we find $R_{\mathrm{d}} \simeq 6.7$ and $24 R_{\mathrm{d}}^{3} \varphi / \pi \simeq 234 \gg 1$. Moreover we find $t_{\mathrm{s}} \simeq$ $1.5 \times 10^{7} \mathrm{~s}$, which is much larger than the steady state value for $T_{1 / 2}$, being $6.4 \times 10^{4} \mathrm{~s}$. The steady state assumption is thus completely unjustified for particles of this size, and the actual initial flocculation rate will be much larger than calculated from the steady state relations.

\section{Conclusions}

The assumption generally made that a flocculation process reaches a steady state very rapidly is invalid when a long range attraction between the particles is present. In this paper we have shown that this is the case with

\footnotetext{
+ Svoboda incorrectly uses $\chi=0.02$ in his calculations in [1]
}

magnetic flocculation of $\mathrm{Mn}_{2} \mathrm{O}_{3}$ colloids, containing particles with a diameter of about $0.8 \mu \mathrm{m}$. A modified version of von Smoluchowski's model for rapid flocculation is presented, in which the capture radius is field-dependent. This model describes the qualitative features of the experimental results quite well, and particularly suggests that the deviations from the 'steady state' increase very rapidly with increasing particle size. Our observation that these effects are already visible for a common paramagnetic material like $\mathrm{Mn}_{2} \mathrm{O}_{3}$ with a particle size below $1 \mu \mathrm{m}$ thus indicates that application of the steady state results will almost always be incorrect for many practical applications of magnetic flocculation, for example in the mining industry, where minerals with particle sizes well above $1 \mu \mathrm{m}$ are treated.

\section{Acknowledgments}

Part of this work has been supported by the Stichting voor Fundamenteel Onderzoek der Materie (FOM) and the Stichting voor de Technische Wetenschappen (STW), with financial support from the Nederlandse Organisatie voor Wetenschappelijk Onderzoek (NWO).

\section{References}

[1] Svoboda J 1982 IEEE Trans. Magn. MAG-18 796

[2] Kruyt H R (ed) 1952 Colloid Science part I (Amsterdam: Elsevier)

[3] Sonntag H and Strenge K 1987 Coagulation Kinetics and Structure Formation (New York: Plenum)

[4] Friedlander S K 1977 Smoke, Dust and Haze (New York: Wiley)

[5] von Smoluchowski M 1917 Z. Phys. Chem. 92129

[6] Fuchs N A 1934 Z. Phys. 89736

[7] Janssen J J M, Baltussen J J M, van Gelder A P and Perenboom J A A J $1990 \mathrm{~J}$. Phys. D: Appl. Phys. 23

[8] Arfken G 1985 Mathemical Methods for Physicists (New York: Academic) ch 17 